\title{
Border sharpness of scar tissue after myocardial infarction as determined by self-navigated free-breathing isotropic 3D whole-heart inversion recovery magnetic resonance
}

\author{
Tobias Rutz ${ }^{2 *}$, Giulia Ginami ${ }^{2,4}$, Davide Piccini ${ }^{2,3}$, Jérôme Chaptinel ${ }^{2,4}$, Simone Coppo ${ }^{2,4}$, Gabriella Vincenti ${ }^{1}$, \\ Matthias Stuber ${ }^{2,4}$, Juerg Schwitter ${ }^{1}$
}

From 19th Annual SCMR Scientific Sessions

Los Angeles, CA, USA. 27-30 January 2016

\section{Background}

The border zone of myocardial scar after myocardial infarction (MI) plays an important role for arrhythmia formation. For this reason, high-resolution 3D information of scar tissue for planning of electrophysiological interventions after MI is highly desirable. This study evaluates sharpness of the borders (SB) of scar after MI by a self-navigated isotropic 3D free-breathing wholeheart magnetic resonance with inversion recovery (3DSN-IR) in comparison to a standard 2D inversion recovery sequence.

\section{Methods}

Patients after MI detected by 2D late gadolinium enhancement (2D LGE) on a standard 2D inversion recovery sequence (resolution $1.3 \mathrm{~mm}^{2}, 8 \mathrm{~mm}$ slice thickness) underwent 3DSN-IR on a $1.5 \mathrm{~T}$ cardiac magnetic resonance scanner (MAGNETOM Aera, Siemens). Data acquisition was performed during the most quiescent systolic phase with a prototype 3D radial trajectory with self-navigation [1] after administration of $0.2 \mathrm{mmol} / \mathrm{kg}$ of Gadobutrol. A non-selective IR pulse was added prior to each acquired k-space segment to the segmented, ECG-triggered, fat-saturated radial SSFP imaging sequence with an isovolumetric resolution of $1.15 \mathrm{~mm}^{3}$. Inversion time was assessed with a $2 \mathrm{D}$ radial scout scan prior to 3DSN-IR. To determine SB, a customized software was used to calculate signal intensity

${ }^{1}$ Division of Cardiology and Cardiac MR Center, University Hospital of Lausanne, Lausanne, Switzerland

Full list of author information is available at the end of the article gradients between two regions [2]. SB in $\mathrm{mm}^{-1}$ of borders "blood pool to scar", "blood pool to non-infarcted myocardium" and "scar to non-infarcted myocardium" were compared between a 2D LGE short-axis slice with $8 \mathrm{~mm}$ slice thickness and two corresponding reconstructed 3DSN-IR short-axis slices, one with isovolumetric voxel size $\left(1.15 \mathrm{~mm}^{3}\right)$ and the second interpolated to $8 \mathrm{~mm}$ slice thickness, all at the same anatomical location.

\section{Results}

Thirteen patients ( 5 females, $58 \pm 10 \mathrm{y}$, time between 2D LGE and 3D LGE $59 \pm 64$ days) were included. All scars visualized by 2 D LGE could be identified by 3DSN-IR. SB was significantly better in 3DSN-IR compared to 2D LGE for the borders "blood pool to non-infarcted myocardium" and "scar to non-infarcted myocardium". There was a trend to a better SB for 3DSN-IR images for the border "blood pool to scar" (see table and figure).

\section{Conclusions}

High resolution 3DSN-IR improves delineation of myocardial scar after MI as expressed by increased border sharpness in comparison to 2D LGE.

\footnotetext{
Authors' details

${ }^{1}$ Division of Cardiology and Cardiac MR Center, University Hospital of Lausanne, Lausanne, Switzerland. ${ }^{2}$ Center for Biomedical Imaging (CIBM) \& Center for Cardiovascular Magnetic Resonance Research (CVMR), University of Lausanne, Lausanne, Switzerland. ${ }^{3}$ Advanced Clinical Imaging Technology, Siemens Healthcare IM BM PI, Lausanne, Switzerland. ${ }^{4}$ Department of Radiology, University Hospital and University of Lausanne, Lausanne, Switzerland.
} 
Table 1 Border sharpness of 2D LGE and 3DSN-IR images. Border sharpness of "blood pool to non-infarcted myocardium"," blood pool to scar" and "non-infarcted myocardium to scar" in mm-1. Data are mean \pm standard deviation or range (interquartile) where appropriate.

\begin{tabular}{|c|c|c|c|c|}
\hline & 2D LGE & 3DSN-IR isovolumetric voxel $(1.15 \mathrm{~mm})$ & 3DSN-IR $8 \mathrm{~mm}$ slice thickness & $\mathbf{p}$ \\
\hline Blood pool - non-infarcted myocardium & $0.029(0.022,0.058)^{*}$ & $0.067(0.04,0.095)$ & $0.071(0.051,0.10)$ & 0.037 \\
\hline Blood pool - scar & $0.083 \pm 0.056$ & $0.121 \pm 0.070$ & $0.124 \pm 0.070$ & 0.176 \\
\hline Scar - non-infarcted myocardium & $0.079 \pm 0.034 \dagger$ & $0.171 \pm 0.086$ & $0.172 \pm 0.074$ & $<0.001$ \\
\hline
\end{tabular}

${ }^{*} p=0.0112 \mathrm{D}$ LGE to $3 \mathrm{DSN}-\mathrm{IR} 8 \mathrm{~mm}$ slice thickness, $+\mathrm{p}<0.006$ 2D LGE vs. 3DSN-IR isovolumetric voxel and 3DSN-IR 8 mm slice thickness

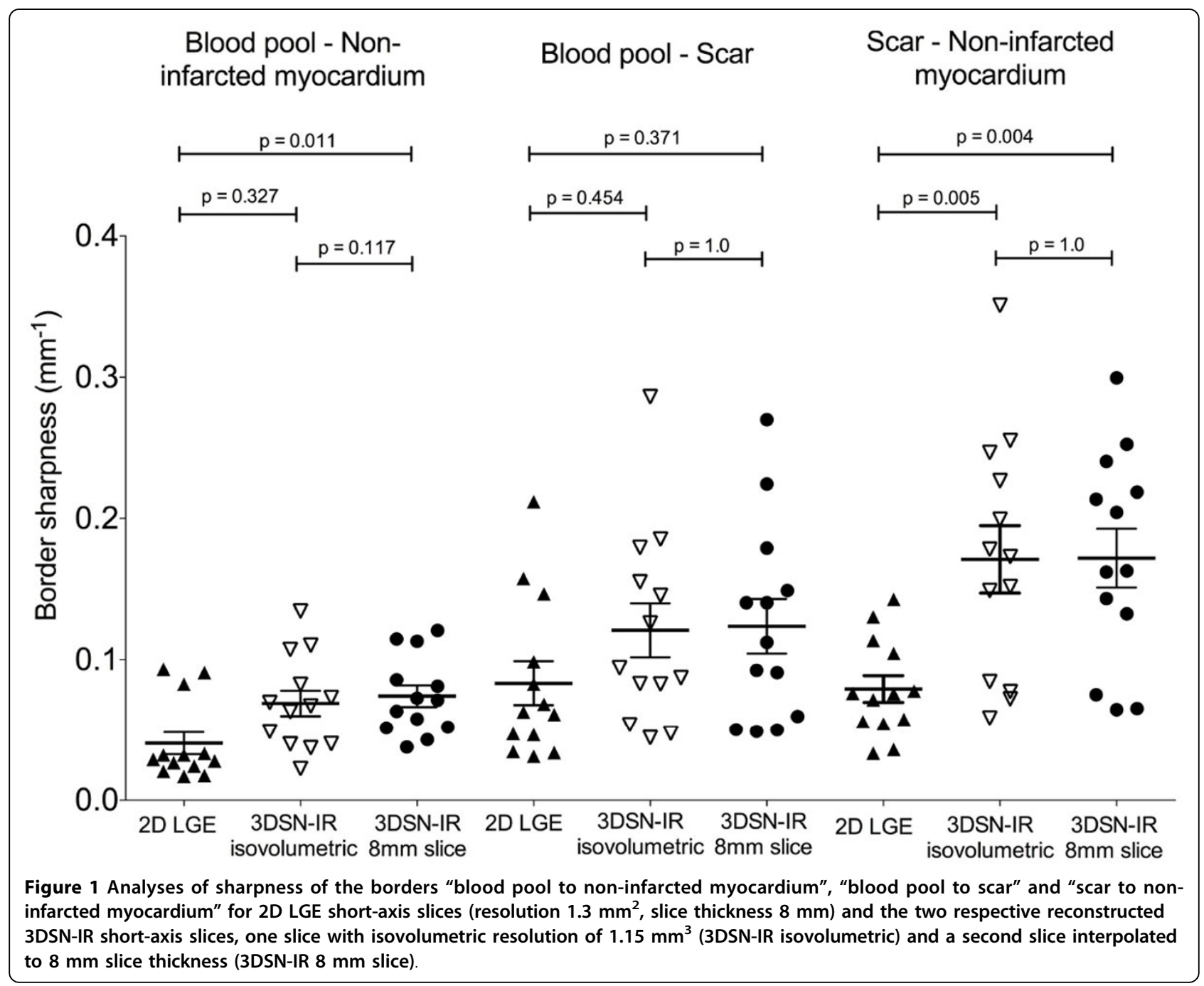

Published: 27 January 2016

\section{References}

1. Piccini $\mathrm{D}$, et al: Respiratory self-navigation for whole-heart bright-blood coronary MRI: methods for robust isolation and automatic segmentation of the blood pool. Magn Reson Med 2012, 68(29):P75-9.

2. Kording F, et al: Doppler ultrasound compared with electrocardiogram and pulse oximetry cardiac triggering: A pilot study. Magn Reson Med 2014.

\section{doi:10.1186/1532-429X-18-S1-P74}

Cite this article as: Rutz et al:: Border sharpness of scar tissue after myocardial infarction as determined by self-navigated free-breathing isotropic 3D whole-heart inversion recovery magnetic resonance. Journal of Cardiovascular Magnetic Resonance 2016 18(Suppl 1):P74. 